Www.jmscr.igmpublication.org

Index Copernicus Value: 79.54

ISSN (e)-2347-176x ISSN (p) 2455-0450

crossrefDOI: https://dx.doi.org/10.18535/jmscr/v7i2.40

\title{
Study of Lipid profile in pulmonary TB cases: pre and post anti-tuberculosis treatment
}

\author{
Authors \\ Dr Vibha Sushilendu ${ }^{1}$, Dr Narendra Kumar ${ }^{2}$, Dr Uday Kumar ${ }^{3}$ \\ ${ }^{1}$ SR, Biochemistry Dept., IGIMS \\ ${ }^{2}$ SR, Dept. of Gen. Medicine, NMCH \\ ${ }^{3}$ Prof. and HOD, Biochemistry Dept, IGIMS
}

\begin{abstract}
Tuberculosis is a major public health problem where India accounts for one fifth of the global burden of $T B$ incident cases. Changes in blood lipid profile has been observed during the natural course of TB infection. Decrease in the level of both TC and HDL cholesterol has been observed in the early stages. This study was undertaken to study the lipid profile of newly diagnosed pulmonary TB patients, to compare it with healthy control and to subsequently study the effect of ATT on lipid profile. 50 newly diagnosed pulmonary TB patients and 50 age and sex matched healthy controls were selected for this study. All the lipid parameters were found to be significantly low in the pulmonary TB infected cases compared to control, at the time of diagnosis. After the completion of ATT, all the lipid parameters were found to increase from the initial value. Recovery from TB after successful ATT is accompanied by improvement of lipid parameters.

Keywords: pulmonary TB, ATT, lipid profile.
\end{abstract}

\section{Introduction}

Tuberculosis (TB) caused by the tubercle bacillus, Mycobacterium tuberculosis (MTB), is a potentially fatal, contagious disease which still remains a major global health care issue ${ }^{[1]}$. It is mainly a lung disease where it forms localized infection after inhalation. TB can however affect any part of the body.

Almost one third of the world's population is infected with Mycobacterium tuberculosis with the majority of these individuals living in less developed countries (WHO, Global Tuberculosis Control, 2011). Worldwide, TB is responsible for more than 1.5 million deaths every year. India is a nation, which is both endemic and epidemic to tuberculosis. Tuberculosis is a major public health problem where India accounts for one fifth of the global burden of TB incident cases. Almost 40 percent of the population in India is infected with the TB bacilli. It is the 2nd leading cause of death killing 2 million people each year ${ }^{[2-5]}$.

Cholesterol, mainly because of its involvement in cardiovascular diseases, has received much attention in recent years. Current recommendations for treatment are aimed at reducing the serum cholesterol levels. However, there are increasing evidences that indicate a link between low blood cholesterol levels and a number of human diseases including tuberculosis. It is reported that low total cholesterol level promotes 
the development of TB whereas hypercholesterolemia confers some protection against infection with Mycobacterium tuberculosis. Despite the existence of such links between cholesterol and $\mathrm{TB}$, it is not known as to which extent the treatment of the disease affects lipid profile in patients with $\mathrm{TB}^{[6-9]}$.

Changes in blood lipid profile has been observed during the natural course of TB infection. Decrease in the level of both TC and HDL cholesterol has been observed in the early stages with increase in TG level later ${ }^{[10]}$. Decreased levels of HDL is a well-recognized independent risk factor for adverse cardiovascular outcomes and this has even been shown to be true in TBinfected individuals, irrespective of other risk factors $^{[11]}$.

This study was undertaken to study the lipid profile of newly diagnosed pulmonary TB patients to compare it with healthy control and to subsequently study the effect of ATT on lipid profile.

\section{Material and Methods}

50 newly diagnosed pulmonary TB patients were selected for this study.50 age and sex matched healthy controls with no complaints and no known diseases were taken as controls. Sputum and blood samples were systematically collected from all patients with clinical signs of pulmonary TB. Patients co-infected with MTB and HIV, and those who underwent an incomplete TB treatment were excluded from the study. Furthermore, patients who were on antibiotic therapy or using any antilipidemic medication, were also excluded from the study. The control group was tested negative upon TB diagnostic testing.

All the lipid profile and other biochemical parameters like glucose, urea, creatinine, AST-
ALT were estimated on fully automated analyzer. Lipid parameters were measured in 50 experimental subjects suffering from pulmonary TB and 50 control subjects. In TB patients, the same individuals were tested before treatment and at the end of treatment. Blood samples were collected after an overnight fast. Anthropometric measurements like body weight and BMI were taken.

Informed written consent was voluntarily obtained from each participant before entering the study.

Data were evaluated by Student's t-test. Quantitative data are expressed as mean \pm SD. $p$ value $<0.05$ is considered statistically significant.

\section{Results}

There were 33 males and 17 females in the TB infected group while the control group included 36 males and 14 females. The mean age of case and control groups were 31.6 years and 33.2 years respectively. All the lipid parameters were found to be significantly low in the pulmonary TB infected cases compared to control, at the time of diagnosis. Total cholesterol and HDL were $130 \pm$ $25 \mathrm{mg} / \mathrm{dl}$ and $34 \pm 7 \mathrm{mg} / \mathrm{dl}$ respectively. Similarly LDL and TG were $70 \pm 14 \mathrm{mg} / \mathrm{dl}$ and $134 \pm 39$ $\mathrm{mg} / \mathrm{dl}$. The value of these parameters in the control group were $183 \pm 32 \mathrm{mg} / \mathrm{dl}(\mathrm{TC}), 46 \pm 9$ $\mathrm{mg} / \mathrm{dl}$ (HDL), $110 \pm 37 \mathrm{mg} / \mathrm{dl}$ (LDL) and $156 \pm 28$ $\mathrm{mg} / \mathrm{dl}$ (TG).

After the completion of ATT of six months duration, all the lipid parameters were found to increase from the initial value. There was significant increase in total cholesterol, HDL and LDL. Triglyceride value, though increased, but the change was not significant. The lipid level after treatment were $150 \pm 40 \mathrm{mg} / \mathrm{dl}$ (TC), $41 \pm 8$ $\mathrm{mg} / \mathrm{dl}(\mathrm{HDL}), 76 \pm 15 \mathrm{mg} / \mathrm{dl}$ (LDL) and $138 \pm 42$ $\mathrm{mg} / \mathrm{dl}$ (TG).

Table 1 Comparative study of lipoid profile $(\mathrm{mg} / \mathrm{dl})$ in cases before ATT and control.

\begin{tabular}{|l|c|c|c|}
\hline Lipid profile & Pre ATT & control & p value \\
\hline TC & $130 \pm 25$ & $183 \pm 32$ & $<0.001$ \\
\hline HDL & $34 \pm 7$ & $46 \pm 9$ & $<0.001$ \\
\hline LDL & $70 \pm 14$ & $110 \pm 37$ & $<0.001$ \\
\hline TG & $134 \pm 39$ & $156 \pm 28$ & $<0.002$ \\
\hline
\end{tabular}


Table 2 Comparative study of lipid profile $(\mathrm{mg} / \mathrm{dl})$ in cases after ATT completion and control.

\begin{tabular}{|l|c|c|c|}
\hline Lipid profile & Post ATT & control & p value \\
\hline TC & $150 \pm 40$ & $183 \pm 32$ & $<0.001$ \\
\hline HDL & $41 \pm 8$ & $46 \pm 9$ & 0.004 \\
\hline LDL & $76 \pm 15$ & $110 \pm 37$ & $<0.001$ \\
\hline TG & $138 \pm 42$ & $156 \pm 28$ & 0.01 \\
\hline
\end{tabular}

Table 3 Comparative study of lipid profile $(\mathrm{mg} / \mathrm{dl})$ in cases before and after completion of 6 months ATT.

\begin{tabular}{|l|c|c|c|}
\hline Lipid profile & Pre ATT & Post ATT & p value \\
\hline TC & $130 \pm 25$ & $150 \pm 40$ & 0.003 \\
\hline HDL & $34 \pm 7$ & $41 \pm 8$ & $<0.01$ \\
\hline LDL & $70 \pm 14$ & $76 \pm 15$ & 0.04 \\
\hline TG & $134 \pm 39$ & $138 \pm 42$ & 0.6 \\
\hline
\end{tabular}

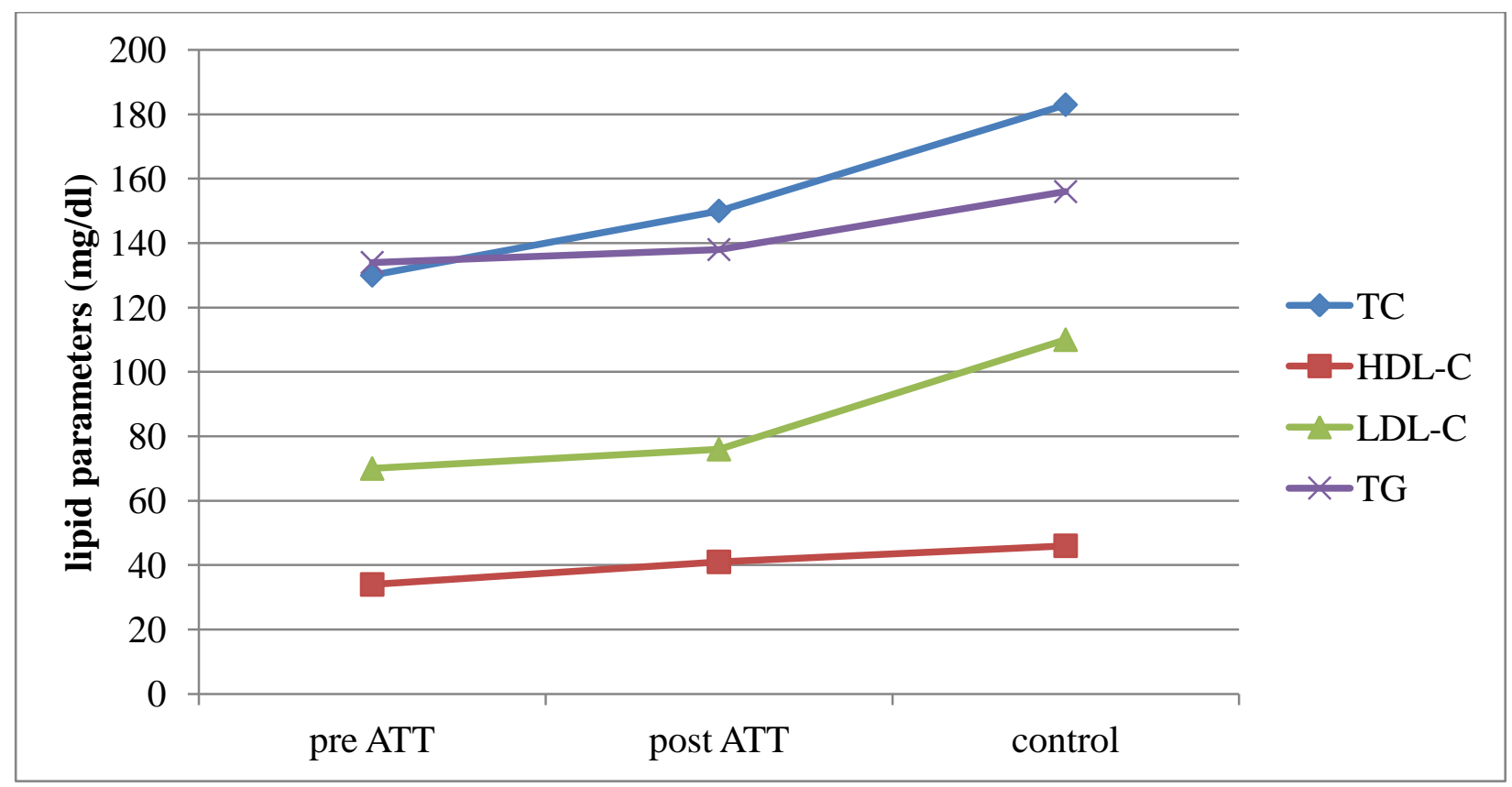

Fig.1 Different lipid fractions in control and cases (both before and after ATT)

\section{Discussion}

Before treatment, TC, HDL and LDL levels were significantly lower in TB patients as compared to normal subjects. At the end of treatment of six months, the levels of TC remained significantly lower in TB treated patients when compared to the healthy controls. However, compared with the levels before treatment, TC and HDL levels increased significantly after treatment .Similar results were obtained by Taparia P. Et al. In their study, all the lipid parameters were significantly $(\mathrm{p}<0.05)$ low in both newly diagnosed and relapse cases of Pulmonary Tuberculosis (PTB) than controls.

Around one third of worlds' population is infected with MTB, but only a small fraction of this develops active disease. Therefore, some specific condition must be there which predisposes these individuals to develop active TB disease ${ }^{[12]}$.

Lipids are important determinants of nutritional status of the body. Low level of lipids in the body specially cholesterol, has been found to increase susceptibility to various infections including TB. Cholesterol constitute a major portion of the lipid content of cell mambranes. It is essential for maintaining membrane fluidity. Even the macrophages recquire cholesterol for their phagocytic activity like cell motility, exocytosis and endocytosis. In cholesterol deficiency, its phagocytic activity is deranged ${ }^{[13-17]}$.

Studies have indicated preferential utilization of fatty acids over carbohydrates by Mycobacterium 
tuberculosis. Hence, during chronic infection, cholesterol utilization by tubercle bacilli might decrease the host pool. Decreased rate of production and higher catabolism during chronic infection may also be the cause of low cholesterol level in TB patients ${ }^{[18]}$.

In this study, the level of TC was significantly lower in patients with TB. Similar observations were reported by Deniz et al and Perez-Guzman et al. An adequate level of cholesterol is necessary for the proper functioning of the immune system against infection. Perez-Guzman et al. have shown that a cholesterol-rich diet accelerates bacteriologic sterilization in patients with TB.

It was found in this study that HDL level was decreased significantly in non-treated TB patients compared to controls, and increased significantly after treatment. Similar results were obtained by Akpovid et al.

During inflammation, catabolism of HDL increases. There is overexpression of inflammatory proteins such as phospholipase A2 and circulating amyloid A, during acute phase of TB. This overexpression is in response to inflammation, which stimulates HDL catabolism. Therefore in successfully treated TB patients, HDL level is supposed to increase after ATT ${ }^{[19]}$.

\section{Conclusion}

Recovery from TB after successful ATT is accompanied by significant improvement of lipid parameters such as cholesterol and HDL. Additional research is required to more fully assess the link between TB treatment and levels of total cholesterol and other lipid components in patients with pulmonary TB.

\section{References}

1. Gemma H, David RT. Vitamin D and Tuberculosis.JPMI.2011;25(3):185-7.

2. M. Muthraj, S. Kamatchiyammal, B. Usharani, S. Manupriya, AR Niranjana. Serum zinc, Calcium and albumin levels in Pulmonary Tuberculosis co-infected with
HIV. Global $\mathbf{J}$ of Biotechnology and Biochemistry.2010;5(1):27-35.

3. Jeon CY, Murray MB. Diabetes Mellitus Increases the Risk of Active Tuberculosis: A Systematic Review of 13 Observational Studies. www. plosmedicine.org. 2008;5 (7):e152.

4. Balasubramanian R, et al. Evaluation of an intermittent six-month regimen in new pulmonary tuberculosis patients with diabetes mellitus. Indian $\mathrm{J}$ Tuberc. 2007;54(4):168-76

5. Anthony D. Harries et al, Defining the research agenda to reduce the joint burden of disease from Diabetes mellitus and Tuberculosis. Tropical Medicine and International Health. 2010;15(6):659-63.

6. Perez-Guzman C, Vargas MH, Quinonez F, Bazavilvazo N, Aguilar A (2005). A cholesterol-rich diet accelerates bacteriologic sterilization in pulmonary tuberculosis. Chest 127:643-651.

7. Wilson RF, Barletta JF, Tyburski JG (2003). Hypocholesterolemia in sepsis and critically ill or injured patients. Crit. Care 7:413-414.

8. Smith GD, Shipley MJ, Marmot MG, Rose G (1992). Plasma cholesterol and mortality: The Whitehall Study. JAMA 267:70-76.

9. Deniz O, Tozkoparan E, Yaman H, Cakir E, Gumus S, Ozcan O, Bozlar U, Bilgi C, Bilgic H, Ekiz K (2006). Serum HDL-C levels, log (TG/HDL-C) values and serum total cholesterol/HDL-C ratios significantly correlate with radiological extent of disease in patients with community-acquired pneumonia. Clin. Biochem. 39:287-292.

10. Bifani, P. J., Plikaytis, B. B., Kapur, V., Stockbauer, K., Pan, X., Lutfey, M. L., Moghazeh, S. L., Eisner, W., Daniel, T. M., Kaplan, M. H., Crawford, J. T., Musser, J. M., and Kreiswirth, B. N. (1996) JAMA 275, 452-457

11. Iseki K, Yamazato M, Tozawa M, Takishita S. (2002): Hypocholesterolemia is a significant predictor of death in a cohort of 
chronic hemodialysis patients. Kidney Int.; 61: 1887- 1893.

12. Mohamed M. Metwally and Hesham Abdel Raheem. Lipid profile in Tuberc-ulosis Patients: A preliminary Report.Life Science Journal.2012;9(1): 719-722.

13. Akiibinu MO, Arinola OG, Ogunlewe JO and Onih EA. Non-enzymatic Antioxidant and Nutritional profile in Newly Diagnosed Pulmonary Tuberculosis Patients in Nigeria. African J. of Biomedical Research.2007; 10: 223-228.

14. Volpato S, Palmieri E, Fellin R, Zuliani G. Acute phase markers are associated with reduced plasma lipid concentrations in a population of hospitalized elderly patients. Gerontology. 2000. 46:22-7.

15. Devlin TM (1992): Biological membranes: structure and membrane transport. In:Textbook of biochemistry with clinical correlation. New York, NY: John Wiley and sons; 226-236

16. Gatfield J, Pieters J. Essential role for cholesterol in entry of mycobacteria into macrophages. Science. 2000; 288:16471650.

17. Maurine D Miner, Jennifer C Chang, Amit K Pandey, Christopher M Sassetti and David R Sherman.Role of cholesterol in Mycobacterium tuberculosis infection.2009; 47:407-11.

18. Maurine D Miner, Jennifer C Chang, Amit K Pandey, Christopher M Sassetti and David R Sherman.Role of cholesterol in Mycobacterium tuberculosis infection. 2009;47:407-11.

19. Taparia P. et al Study of lipid profile in pulmonary tuberculosis patients and relapse cases in relation with disease severity - A pilot study International Journal of Sciences \& Applied Research , 2(1), 2015; 41-50. 